\title{
Improved clay mineral identification by LIBS/Raman data fusion: implications for in-situ Mars exploration
}

\author{
ERIN GIBBONS ${ }^{1 *}$, RICHARD LEVEILLE ${ }^{1}$, AND KIM \\ BERLO $^{1}$ \\ ${ }^{1}$ Department of Earth and Planetary Sciences, McGill \\ University, Canada (*correspondence: \\ erin.gibbons@mail.mcgill.ca)
}

We introduce a new methodological approach which uses Raman spectroscopy and laser-induced breakdown spectroscopy (LIBS) to classify impure clay-rich geological specimens according to their dominant mineralogy. Raman and LIBS provide complementary information about the molecular structure and elemental composition of an interrogated target, and, when considered simultaneously, contribute to a more comprehensive characterization of the system under study. To capitalize on this synergy, Raman and LIBS data were collected from various clay-rich specimens and concatenated into a single unique spectral identifier of specimen composition - an approach known as low-level data fusion (DF). Multivariate analyses, including principal component analysis (PCA) and linear-discriminant analysis (LDA), were used to build discrimination models and classify the specimens based on their predominant clay mineralogy. To quantify our results, we define classification accuracy as the percentage of the number of correctly classified samples to the total number of analyzed specimens within each identified mineral group. We compared the classification accuracy achieved by the fused data sets compared to that obtained by standalone use of Raman and LIBS data.

We found that the LIBS data achieved $96 \%$ correct classification of the specimens. Our Raman data were influenced by impurity phases that masked the weak Raman signature of the clay minerals, resulting in $93 \%$ classification accuracy. Perfect classification (100\% accuracy) of the specimens was attained via the low-level DF strategy. Thus, the results of our pilot study indicate that DF has the capacity to improve the classification of clay-rich geological specimens by their modal mineralogy and highlights the importance of considering innovative approaches to the management of data resources to maximize scientific output.

LIBS and Raman will be available on NASA's Mars 2020 rover and suggest that the synergistic use of their spectra may improve the identification of clays on Mars. Given that clay minerals are a robust indicator of past water activity, this capability could help direct astrobiological exploration efforts. Research into this topic is underway. 